\title{
Correction To: Aspergillus fumigatus-Stimulated Human Corneal Epithelial Cells Induce Pyroptosis of THP-1 Macrophages by Secreting TSLP
}

\author{
Qingshan Ji, ${ }^{1}$ Lisong Wang, ${ }^{1}$ Jiajia Liu, ${ }^{1}$ Yali Wu, ${ }^{1}$ Huayi Lv, ${ }^{1}$ Yuechun Wen, ${ }^{1}$ Lei Shi, ${ }^{1,2,5}$ \\ Bin $\mathrm{Qu},{ }^{3}$ and Nóra Szentmáry ${ }^{2,4}$
}

Correction to: Inflammation (2021) 44(2):682-692

https://doi.org/10.1007/s10753-020-01367-x

The original publication of this article contained mistakes.

The corresponding affiliation should be:

To whom correspondence should be addressed at Department of Ophthalmology, The First Affiliated Hospital of USTC, Division of Life Sciences and Medicine, University of Science and Technology of China, No. 17, Lujiang Road, Hefei, 230001, Anhui, China. E-mail: drshilei@sina.cn

The online version of the original article can be found at https://doi.org/ 10.1007/s10753-020-01367-x

\footnotetext{
${ }^{1}$ Department of Ophthalmology, The First Affiliated Hospital of USTC, Division of Life Sciences and Medicine, University of Science and Technology of China, No. 17, Lujiang Road, Hefei, 230001, Anhui, China

${ }^{2}$ Dr. Rolf M. Schwiete Center for Limbal Stem Cell and Congenital Aniridia Research, Saarland University, Kirrberger Strasse 100 Geb. 22, 66424 Homburg, Saarland, Germany

${ }^{3}$ Biophysics Center for Integrative Physiology and Molecular Medicine (CIPMM), Saarland University, Kirrberger Strasse 100 Geb. 48, 66421 Homburg, Saarland, Germany

${ }^{4}$ Department of Ophthalmology, Semmelweis University, Budapest, Hungary

${ }^{5}$ To whom correspondence should be addressed at Department of Ophthalmology, The First Affiliated Hospital of USTC, Division of Life Sciences and Medicine, University of Science and Technology of China, No. 17, Lujiang Road, Hefei, 230001, Anhui, China. E-mail: drshilei@sina.c
} 